\title{
Successfully Tracheostomy and Monobloc Advancement with External Frontofacial Distraction Followed by Simultaneous Adenoidectomy, Conchotomy, and Septoplasty in Severe Late Crouzon Syndrome
}

\author{
Diar Mia Ardani ${ }^{1}$, Magda Rosalina Hutagalung ${ }^{2}$, Irwan Kristyono ${ }^{3}$ \\ ${ }^{1}$ Medical Staff, Pharyngeal Larynx Division, Department of Otorhinolaryngology-Head and Neck Surgery, Faculty \\ of Medicine, Universitas Airlangga-Dr. Soetomo General Academic Hospital, Surabaya, Indonesia, ${ }^{2}$ Medical \\ Staff, Department of Plastic Reconstructive and Aesthetic Surgery, Faculty of Medicine, Universitas Airlangga-Dr. \\ Soetomo General Academic Hospital, Surabaya, Indonesia, ${ }^{3}$ Medical Staff, Department of Otorhinolaryngology- \\ Head and Neck Surgery, Faculty of Medicine, Universitas Airlangga-Dr. Soetomo General Academic Hospital,
} Surabaya, Indonesia

\begin{abstract}
A 10-year-old male patient presented with Severe Late Crouzon Syndrome, with turri-brachycephaly, and severe exorbitism of both eyes, visual loss, beaked nose and maxilla hypoplasia. The head circumference was $54 \mathrm{~cm}$ and 90 precentile. Patient had a high arched palate, tonsil hypertrophy and type III malocclusion. From the multidisciplinary meeting, in the first stage it was decided to first secure the airway with Tracheostomy then immediately followed by Monobloc Advancement with External Frontofacial Distraction. Then in the second stage, after removal of the rigid external distractor, by 9 months after first surgery, the patient underwent a simultaneous procedures of Adenoidectomy, Conchotomy, and Septoplasty. Thus, after patient got stable in follow-up, Nasendoscopy and Tracheostomy devices was removed. After 11 months follow-up. Visual function still not getting better. However turri-brachycephaly, exorbitism of both eyes, beaked nose and maxilla hypoplasia, head circumference, arched palate, tonsil hypertrophy and the malocclusion were getting much reduced, and the patient getting happier and more active.
\end{abstract}

Keyword: Adenoidectomy, conchotomy, septoplasty, crouzon syndrome.

\section{Introduction}

Crouzon syndrome is a congenital abnormality in the fetus in the womb during organ formation and is a dominant autosomal disorder. This disease was first described by neurosurgeon Octave Crouzon from

\section{Corresponding Author:}

Diar Mia Ardani

Pharyngeal Larynx Division, Department of

Otorhinolaryngology - Head and Neck Surgery, Faculty of Medicine, Universitas Airlangga - Dr. Soetomo General Academic Hospital, Jalan Mayjen Prof. Dr. Moestopo no. 6-8, Airlangga, Gubeng, Surabaya, East Java 60286, Indonesia e-mail:diarmiaa@gmail.com
France in 1912. This syndrome is classified as a group of syndromes that are rarely found with the main characteristic of craniosinostosis. Craniosinostosis is a condition in which one or more fibrous sutures on the babies cranium merge and harden prematurely, thereby changing the pattern of cranial growth. In the case of Crouzon syndrome organ formation does not develop properly, especially in cranium sutures that close prematurely so that when the head forms it becomes imperfect ${ }^{(1-4)}$.

The four main physical findings that are characteristic of Crouzon's syndrome are exorbitism, retro maxillary, inframaxillism, and paradox retrognathia. The manifestations of Crouzon syndrome that can be found in the oral cavity, namely mandibular prognathy, crowded teeth in the maxilla, maxillary atresia, crossbiteanterior 
with posterior open bite, curvature of the maxillary jaw and cleft palate. Crouzon's syndrome accounts for $4.8 \%$ of all cases of craniosinostosis, with an incidence of 1 : 25,000 to 1: 65,000 births and an autosomal dominant disorder. This pathological variation is believed to be caused by the mutation of the Fibroblast Growth Factor Receptor 2 gene (FGFR2) on chromosome 10q26 $6^{(3,4)}$.

Children with craniosinostosis syndrome including Crouzon syndrome are prone to obstructive sleep apnea (OSA) syndrome with an estimated prevalence of between 40 and $85 \%$. The cause of OSA is mainly caused by anatomical midfacial hypoplasia. Premature stitch fusion causes midfacial hypoplasia, increased nasopharyngeal resistance, and narrowed oropharyngeal space, all together contributing to OSA. Continuous positive airway pressure (CPAP) and surgical techniques are the two main OSA therapies for adults. Children with Crouzon syndrome are faced with more complicated problems including corneal exposure, craniofacial abnormalities, and malocclusion. Surgery for osteogenesis and craniomaxillofacial disorders seems to be a better choice for these children because it can relieve or even complete OSA by correcting midfacial hypoplasia and improving facial profiles ${ }^{(5-7)}$.

The diagnosis of Crouzon syndrome is done by history, physical examination, oral cavity, functional and radiographic analysis. In general, before the treatment of orthodontic anomalies, surgery is needed first. The treatment of Crouzon syndrome patients requires several stages. The first stage involves treating craniosinostosis by correcting its frontal-orbital abnormalities in the sagittal direction. Next stage is reconstruction of the face and so on until it reaches the final stage of reconstruction which is a class III dental malocclusion treatment ${ }^{(8,9)}$.

Case Presentation: Patient was referred from a social organization and presented with turribrachycephaly, severe exorbitism of both eyes, visual loss, beaked nose and maxilla hypoplasia (Figure 1). The head circumference was $54 \mathrm{~cm}$ and 90 precentile (figure 2). Patient had a high arched palate, tonsil hypertrophy and type III malocclusion. Ophthalmologic review revealed decreased vision in both eyes to light perception. Funduscopic examination was not able to be performed due to patient's agitation. Neurosurgical review supported monobloc surgery and based on MRI showed Arnold Chiari malformation type 1. Paediatric review revealed obesity and good development based on IQ, PEDSQL and PSC-17. ENT review revealed adenoid hypertrophy through nasoendoscopy, septal deviation, severe bilateral conductive hearing loss. Psychiatric review showed IQ scored 90. Polisomnography revealed Sleep Apneu (central sleep apnea, obstructive sleep apnea and mixed apnea with severe AHI 74x/hours).

From the multidisciplinary meeting, in the first stage it was decided to first secure the airway with tracheostomy by head and neck surgeon then immediately followed by monobloc advancement with distraction. Then in the second stage, after removal of the rigid external distractor, ENT would do an adenoidectomy, septoplasty and conchotomy. In the final stage, after re-evaluation by ENT and Head and Neck surgeon. Monobloc advancement with RED was strongly indicated due to severe OSA, increased ICP and threatening blindness. It is a standard protocol for Crouzon beyond 8 years of age. We performed the surgeries in stages and only after securing the airway with a tracheostomy. Adenoidectomy was done nine months after the monobloc.

The tracheostomy was removed only after ENT review indicated no contraindication and weaning was successful. He recovered uneventfully. He became active and happy, breathing spontaneously, sleeping well, and his eyes were fully healed eventhough the visual acuity remained at light perception and mastication became more effective. Details of patient development from the beginning to the end of treatment can be seen in figures 3-4.

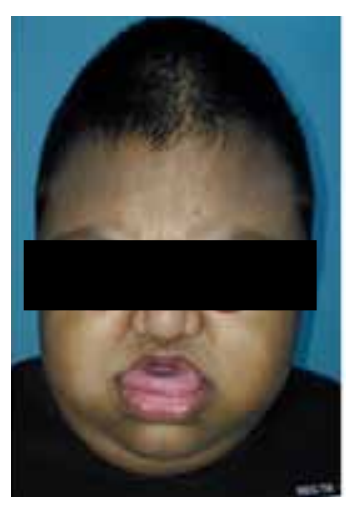

(A)

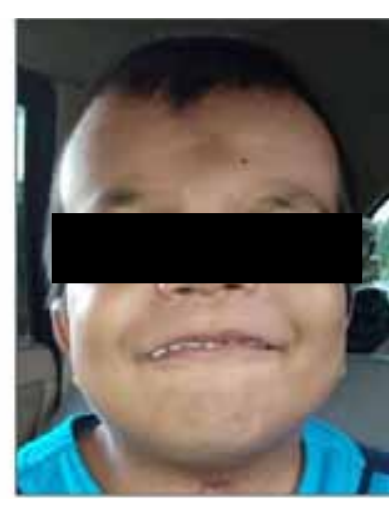

(B)
Figure 1. The patient's condition on pre-surgery (A) and post-surgery (B) 

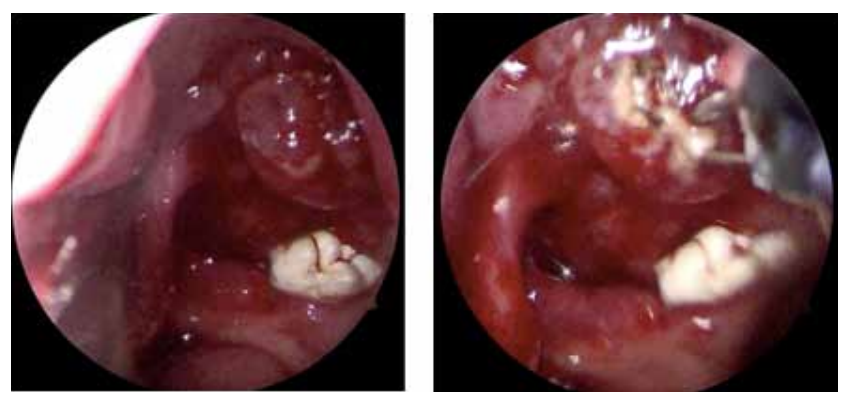

(A)

(B)

Figure 2. Adenoid condition of patients before (A) and after (B) performing radiofrequency

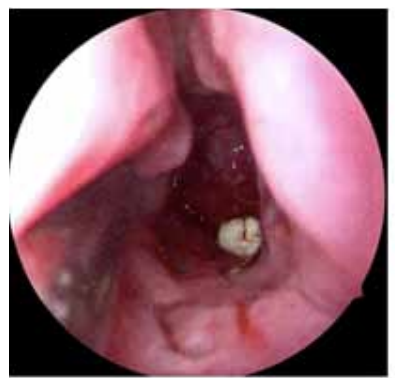

(A)

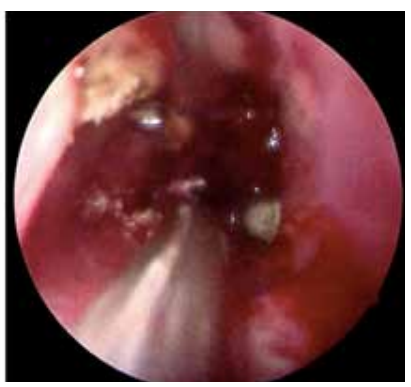

(B)
Figure 3. Right patient turbinate before $(A)$ and after $(B)$ radiofrequency

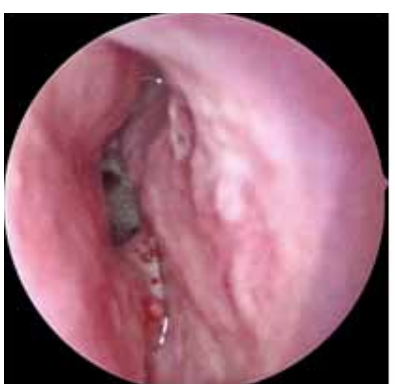

(A)

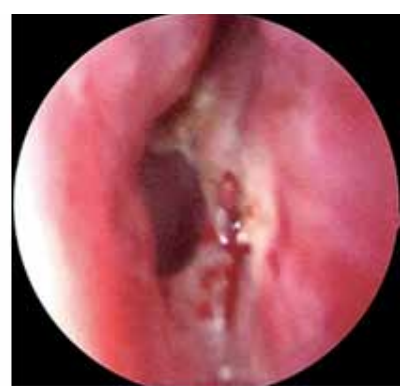

(B)
Figure 4. Left patient turbinate before (A) and after (B) radiofrequency

\section{Discussion}

Sleep apnea syndrome is a syndrome with the discovery of episodes of apnea or hypopnea during sleep. Apnea can be caused by a central disorder, obstructive airway, or a mixture. Obstructive apnea is the cessation of air flow to the nose and mouth even with breathing efforts, while central apnea is the cessation of breathing which is not accompanied by an effort to breath due to the absence of breath stimulation. Obstructive hypoventilation is caused by partial obstruction of air flow which causes hypoventilation and hypoxia. The term obstructive hypoventilation is used to indicate the presence of hypopnea, which means a reduction in air flow $(7,10)$

The term OSAS is used in total or partial airway obstruction syndrome which causes significant physiological disorders with variation clinical impacts. The term primary snoring is used to describe children with snoring habits that are not related to obstructive apnea, hypoxia or hypoventilation. Guilleminault et al. Defined sleep apnea as apnea episodes 30 times or more in 8 hours, for at least 10 seconds and occurred both during the sleep phase of rapid eye movement (REM) and non rapid eye movement (NREM). There are terms of apnea index (AI) and hypopnea index (HI), namely the frequency of apnea or hypopnea per hour. Apnea or hypopnea index can be used as an indicator of the mild severity of OAS ${ }^{(7-9)}$.

Risk factors for OSAS in children include the result of adenoid and tonsillar hypertrophy, craniofacial disproportion, obesity. Adenoid and tonsillar hypertrophy are the conditions that most often cause OSAS in children. Adenoid and tonsil size is not directly proportional to the severity of OSAS. There are quite large children with adenoid hypertrophy, but OSAS that occurs is still mild, other children with mild adenoid enlargement show severe OSAS symptoms. Adenoid and tonsil hypertrophy can also cause complications in children with basic bone disorders. Although in most OSAS children it improves after adenotonsillectomy, a small portion will remain after surgery. In one study a small proportion of children with OSAS who had been successfully treated with adenotonsillectomy surgery then experience recurrence of symptoms during adolescence. Children with craniofacial anomalies that experience a marked narrowing of the airway structure (micrognation and midface hypoplasia) will experience OSAS. In children with craniofacial disproportion can cause airway obstruction even without adenoid hypertrophy $(7,10)$

Another cause of OSAS is obesity. In adult obesity is the main cause of OSAS whereas in obese children it is not the main cause. The mechanism of the occurrence of OSAS in obesity because there is a narrowing of the upper airways due to accumulation of fat tissue in the muscles and soft tissues around the airways, as well as external neck and jaw compression. Determination of obesity can be done by counting body mass index (BMI) and measurement of neck circumference. For OSAS determination, the more important is the neck circumference compared to BMI. It is well known that 
large neck circumference or obesity in the upper area is associated with an increase in cardiovascular disease, as well as allegedly associated with snoring and OSAS. It is suspected that the accumulation of fat in the neck area can make the upper airway more narrow. Another possibility is that obese patients with large necks have velofarings that are more prone to collapse so that it can facilitate the occurrence of upper airway obstruction at bedtime ${ }^{(7,10)}$.

The most common clinical manifestations are difficulty breathing during sleep, which usually progresses slowly. Before symptoms of breathing difficulties occur, snoring is a symptom that initially arises. Snoring in children can occur continuously (every sleep) or only in certain positions. In OSAS, children generally snore every time they sleep with loud snoring coming from outside the room and see episodes of apnea that may end with body movements or wake up. A small number of children do not show classic snoring, but in the form of grunting or breathing, noisy breathing. Breathing efforts can be seen with retraction. The position during sleep is usually on his stomach, half sitting, or neck hyperextension to maintain airway patency $(7,10)$.

On physical examination can be seen mouth breathing, adenoidal facies, midfacial hypoplasia, retrognation, micrognation or other craniofacial abnormalities, obesity, failure to thrive, allergic stigmata such as alergic shiners or horizontal nasal folds. The patency of the nasal passages must be assessed, note the presence of septal deviation or nasal polyps, tongue size, integrity of the palate, orofarings, redundant palatal mucosa, tonsil size, and size of the uvula, may be found pectus excavatum. The lungs are usually normal on auscultation examination. Examination of the heart can show signs of pulmonary hypertension such as increased pulmonary component of heart sound II, right ventricular pulsation. Neorological examination must be performed to evaluate muscle tone and development status ${ }^{(7,10)}$.

OSAS management in children is divided into two major groups, namely surgical and medical (non surgical). Surgical measures performed are tonsillectomy and or adenoidectomy and correction of craniofacial disproportion, while medical therapy can be a diet in obese children and nasal use of CPAP (Continuous Positive Airway Pressure) ${ }^{(7,10)}$.

Many experts argue that the action of tonsillectomy and or adenoidectomy is a necessary action because the benefits are greater. The rate of recovery of this action in children is around $75 \%-100 \%$. In children with etiology of adenoid hypertrophy and tonsils alone the success rate is high but if accompanied by other risks such as obesity and craniofacial disproportion then postoperative OSAS will still occur. However, because OSAS occurs due to the size of the structure of the upper respiratory tract components relative to the absolute size of the tonsils and adenoid, the experts believe that tonsillectomy and or adenoidectomy is still needed in the above conditions. Post tonsillectomy and or adenoidectomy polysomnographic monitoring is needed as a followup. Sometimes symptoms persist and within a few weeks they disappear. Other non-medical treatments such as handling obesity are still carried out despite tonsillectomy and or adenoidectomy $(7,10)$.

\section{Conclusions}

One of the rare cases of Crouzon syndrome which is handled in a multi-disciplinary manner by the person in charge of the reconstructive plastic surgeon, is consulted to the otorhinolaryngology - head and neck surgey with a diagnosis of obstructive sleep apnea. The interventions given were adenoidectomy, conchotomy, and septoplasty which were able to improve the quality of life of patients.

Conflict of Interest: The authors declare that they have no conflict of interest.

Funding: Nothing.

Acknowledgement: We once gave an oral presentation of our paper at the 2019 ICORL event in South Korea (http://www.koreaorlmeeting.org/ workshop/2019spring/org.html) on April 25-28, 2019.

Compliance with Ethical Standards: Authors declare no conflicts of interest. There are no funding sources for the underlying scientific work. This case report was used only for academic purposes and we have obtained the consent of the patient's guardian.

\section{References}

1. Di Rocco F, Arnaud E, Renier D. Evolution in the frequency of nonsyndromic craniosynostosis. Journal of Neurosurgery: Pediatrics. 2009;4(1):21-5.

2. Agochukwu NB, Solomon BD, Muenke M. Impact of genetics on the diagnosis and clinical 
management of syndromic craniosynostoses. Childs Nerv Syst. 2012;28(9):1447-63.

3. Teven CM, Farina EM, Rivas J, Reid RR. Fibroblast growth factor (FGF) signaling in development and skeletal diseases. Genes \& Diseases. 2014;1(2):199213.

4. Ciurea AV, Toader C. Genetics of craniosynostosis: review of the literature. J Med Life. 2009;2(1):517.

5. Zandieh SO, Padwa BL, Katz ES. Adenotonsillectomy for obstructive sleep apnea in children with syndromic craniosynostosis. Plastic and reconstructive surgery. 2013;131(4):847-52.

6. Al-Saleh S, Riekstins A, Forrest CR, Philips JH, Gibbons J, Narang I. Sleep-related disordered breathing in children with syndromic craniosynostosis. Journal of Cranio-Maxillofacial Surgery. 2011;39(3):153-7.
7. Bannink N, Nout E, Wolvius EB, Hoeve HLJ, Joosten KFM, Mathijssen IMJ. Obstructive sleep apnea in children with syndromic craniosynostosis: long-term respiratory outcome of midface advancement. International Journal of Oral and Maxillofacial Surgery. 2010;39(2):115-21.

8. Johnson D, Wilkie AOM. Craniosynostosis. Eur J Hum Genet. 2011;19(4):369-76.

9. Anderson PJ, Evans RD. Re: Metacarpophalangeal analysis in Crouzon syndrome. American journal of medical genetics. 1998;80(4):439-.

10. Driessen C, Joosten KFM, Bannink N, BrederoBoelhouwer HH, Hoeve HLJ, Wolvius EB, et al. How does obstructive sleep apnoea evolve in syndromic craniosynostosis? A prospective cohort study. Archives of Disease in Childhood. 2013;98(7):538-43. 